\title{
Methods of Torque Ripple Minimization techniques on Switched Reluctance Motor
}

\author{
Maheshbabu Valeti, D.Susitra
}

\begin{abstract}
In recent decades, Switched Reluctance Motor (SRM) drive has proved to be an attractive alternate for the conventional industrial drives. Many published research works have proved that SRM drives finds significant application in almost all industrial sectors. This paper presents a comprehensive review on various control strategies of SRM drive implemented in literatures. The objective of this review is to identify the accurate control strategies for controlling the SRM speed and torque. This paper also reviews the various strategies for minimization of the torque ripples, acoustic noise and vibration in SRM
\end{abstract}

Index Terms: PWM Technique, switched reluctance motor, torque ripple,

\section{INTRODUCTION}

SRM is an appropriate choice for variable speed industrial drive applications due to its significant features such as low cost, simple structure, robust construction, high torque, and high speed. SRM has no windings on rotor it has windings on stator side only, due to this the losses in SRM is less compared to other motors. SRM has doubly salient structure so that switched reluctance has high torque ripples this is main drawback in switched reluctance motor. Due to presence of torque ripples unwanted acoustic noise and high vibrations are produced. The torque ripple mainly depends on electric design variables.

In this work, various torque minimization control strategies for SRM drive such as Digital PWM current controller, Fuzzy adaptive direct torque control, Neuro-fuzzy control technique, Speed signal processing, Current Chopping Control, Voltage Chopping Control, Single Pulse Control, Instantaneous Torque Control, Torque Sharing Function and direct Instantaneous Torque Control are analyzed in an elaborated manner. Published literatures on these control algorithms and the implementation results of such techniques are comprehensively reviewed.

Section I presents the digital control method for torque ripple minimization in SRM. In Section II, the fuzzy direct adaptive torque controller is discussed. The effect of fuzzy logic controller on the issue is discussed in section III. Section IV presents the Neuro-fuzzy control technique. Section $\mathrm{V}$ focus about the speed signal processing method.

Revised Manuscript Received on July 05, 2019.

Maheshbabu Valeti, Research scholar, Department Of

Electrical And Electronics Engineering, Sathyabama institute of Science and Technology, Chennai, 600119

D.Susitra, professor, Department Of Electrical And Electronics Engineering, Sathyabama institute of Science and

Technology Chennai,600119
Section VI presents the current control chopping method. In section VII, the direct instantaneous torque control is discussed. Section IX discuss about the torque sharing function.

\section{RELATED WORKS}

Cheng-Yu Lai, et al., the application examines the relationship among the individual's networked disinhibiting behavior and their interest of excitement while using cyberbullying. However, networked disinhibiting can be used to moderately describe the reasons, the interrelations among individual's motivation, unstate condition, and benefit of automation and cyberbullying behavior remain topics that needs advance inquiry [1].Samuel Owusu, et al., this research understands whether teenager's empathy has an impact on their positive bystander behavior and proposed two hypotheses about the effect of empathy due to harassment. The study has to increase sample size, improving the reliability of the affective empathy scale, and investigating possible moderating factors [2].Ziyi $\mathrm{Li}$, et al., an automated cyberbullying identification method that accomplish the parent-child interrelationship among comments in the post to arrest the attitude from the victim to identify cyberbullying comments [3].Arpita Chakraborty, et al., provided a design model that possess a superior semantic understanding of cyberbullying messages and can identify different types of bullying that can occur with/without the presence of profane/abusive words. But it cannot detect the difference profound signals present in bullying messages and responses, i.e., emotional will help in accurately identifying different forms of bullying and foster a deeper understanding of their impact on recipients [4]. Finally, complete content and organizational editing before formatting. Please take note of the following items when proofreading spelling and grammar. Dominic Di Franzo, et al., bystander intervention intimate the false effects of bullying and they accepted various kinds of data around the audience size and viewing notifications designed to enhance a sense of particular authority in bystanders. Although most of the bystanders could not intervene directly, they simply generate reports and send to the Victim [5].Peijun Zhao, et al., examined the correlation between emoticon usages and tweet comfortable, tweet architecture, user enumeration using an mmGRU model for predicting emoticon categories and positions motivated by the observations. This system could not manipulate a list of case histories in display and various types of emoticons are used in social media [6].Chenliang Li,

et al., promoted the linguistically associated words beneath the similar category

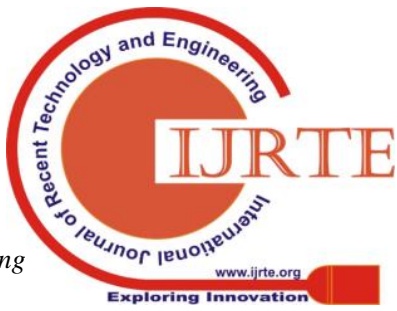


when the fragmented process, by using the Generalized Polya Urn (GPU) model, enlarged the types same for two linguistically associated words that may not occur many times in the short hand text selection. This system does not concentrate more on topic inference strategy and less accuracy in detecting computer short form of words [7].

Mohammadreza Rezvan, et al., contributed both a character comments collection and an abusive word dictionary takes various categories of stalking contented such as, intellectual, appearance - related harassment, political, racial and sexual. The system could not perform analysis on harasser and victim's language [8].Santosh Joshi, et al., construct a machine learning technique for malware identification using Random Forest classifier for the action series information taken from the virtual machine surroundings. It does not include the implementation of additional classification algorithms and performing a comparison to identify the best algorithm for analyzing the process list data structures [9]. Jacob L. Bigelow, et al., elaborates initial conclusions for an application that uses Latent Semantic Indexing (LSI) for the cyberbullying detection in an acknowledged selection of posts from Formspring. Me. This system does not test in other domains, like a collection of SMS messages or Twitter and Facebook posts [10].

Xiang Zhi Tan, et al., explored an efficient approach for robots reduce human being offense: activating bystander interventions. The disadvantages of this application are, need to achieve without permanent guidance in personal surroundings [11]. Lindsay Blackwell, et al., this approach explains the architecture and prediction of HeartMob, a platform constructed by and for the people who are extremely concerned with the most severe online forms of impersonation. Moreover, the platform guidelines and broadcasting apparatus are created for the apparently similar user base and do not explanation for respective one's involvement and applications of social brutality [12]. Jessica Vitak, et al., displayed the ramification of aspect correlate with women's online cyberbullying and point to the demand for both philosophers and engineers to continue their efforts to decrease cyberbllying behaviors and reduce their pessimistic effects. This does not extend the most empathetic of the present state of online annoyance for women and unable to construct more efficient apparatus based on what we already know more about the adults factors combined with intimidation in social media [13].

Noviantho, et al., introduced to build a design for classification with excellent certainty in detecting cyberbully comments using the Support Vector Machine (SVM) and Naive Baye's method. The classification of cyber bullying on text conversations that also contains shorted hand text is not detected. Furthermore, organizing text in the comments are further, denouncing due to text in the comments frequently have shorted hand texts [14].Rahat Ibn Rafiq, et al., this proposed system introduced an eminently extensible without signifying veracity and deeply responsible for establishing signals. This system does not develop a diverse, scalable and responsive classifier with better precision and recall performances for Vine as well other diverse SNS like Facebook [15]. Vivek K. Singh, et al., this system introduces the usage of computerized text and image analysis Application Programming Interface for the multi-modal (textual and visual) cyberbullying identification and descriptions that the achievements of computerized cyber bullying detector enhances with the usage of a multi-modal visual + textual) technique when correlated to a text only technique. In addition, research efforts could leverage the availability of emoticon contents as well as short words for better cyber bullying detection [16]. Elaheh Raisi and Bert Huang aim to improve automated detection of cyber bullying presently advancing weakly supervised techniques that are furthermore evaluate the online countenance or the progression of comments in the post. Although, the detection of an automated cyberbully is the main defect between lots that must be clarified to competently addressed the cyberbullying anomaly [17]. MauroColetto, et al., investigated the end-users who can accept abusive language and antagonism words in Facebook and Twitter and other social media by analyzing their profile pictures. Moreover, aggressive users smile less, and they appear not happy in their profile pictures, dominated by fear, sadness and anger using these particulars the aggressive users cannot able to detect, so it provides less accuracy [18].

Based on the above Literature Survey to overcome the drawbacks of the various data around the audience size and viewing notifications at the time of cyberbullying [5], this proposed system is going to identify a cyberbully word from emoticons and short hand text in the comments section and categorized such as Denigration, Trickery, Flaming and Cyberstalking which helps the society to forbid the cyberbullying victims from facing the troubles of cyberbullying.

\section{Proposed System}

\section{Digital PWM Controller}

SRM is driven by asymmetrical half bridges. Due to this current controller is employed to generate the switching pulses to half bridge based upon current reference. This current controller is given by a speed controller or torque distributor. Hysteresis controller [1] is used for current control which gives better results than the flat chopping, but hysteresis has the drawback of changeable switching frequency and high rate of sampling. The drawback in hysteresis controller is overcome by fixed frequency PWM controllers [4].

Open loop controller that is PI controller is used to overcome those drawbacks, by using PI controller iterative learning control and back EMF compensation is analyzed, and this controller has a drawback of slow response. Digital PWM current controller [5] is used to achieve fast response, accurate tracking, immunity noise, model mismatch and stability. This controller can

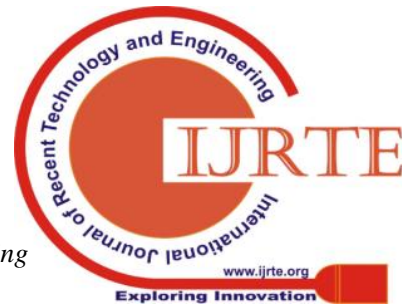


either control the current directly or indirectly by controlling flux linkage.

SRM drive is controlled by digital PWM current controller and this controller is controlled by a control board with a DSP.

SRM drive is controlled by SRM control algorithm and this is implemented in TI'S DSP TMS320F28335

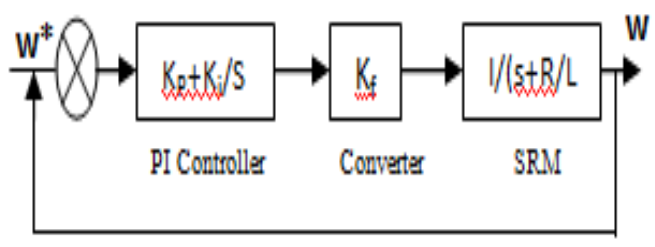

Figure.1 Conventional PWM Controller

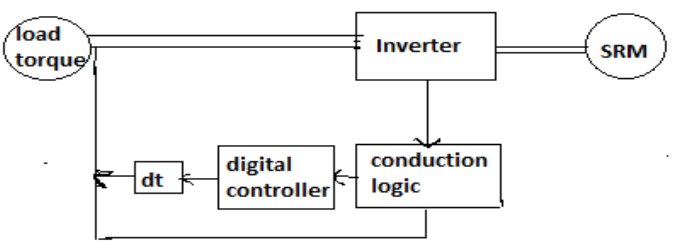

Figure.2 Digital PWM Controller

\section{FuzZy Direct AdAPTIVe TORQUe CONTROLlER}

In [8] discusses the torque ripple minimization using the Fuzzy Direct Adaptive Torque Controller. The torque ripples such as prolonged torque ripples and flux ripples can be reduced by using the hysteresis comparator, but this hysteresis comparator has the drawback of variable switching frequency due this variable switching frequency the performance of the motor will not be improved. Fuzzy Direct adaptive torque controller [8] is used to reduce the flux ripples along with the DTFC. DTFC compares the reference electromagnetic torque and desired stator flux with the estimated values, then we obtain the flux and torque errors and these errors are fuzzily into fuzzy sets.

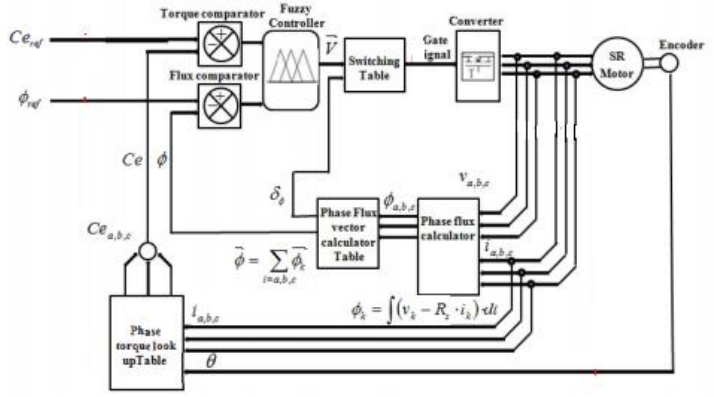

Figure.3 Fuzzy adaptive torque controller

\section{Fuzzy Logic Controller}

In [2] discusses the torque ripple minimization using the fuzzy logic controller. Switched reluctance motor has several advantages but it has the drawback of torque ripples. Previously p-controller, PID-controller is used to reduce the torque ripples but these controllers reduce the ripples up to some extent only. fuzzy logic controller [2] is used to reduce the ripples in the switched reluctance motor. By using FLC we can obtain better results. In this FLC with PI, FLC with PID controller is used to get better results. In this FLC provides compensating current to compensate torque ripples, FLC has two inputs they are reference value of current and rotor position form then the position output is compensated value of current.

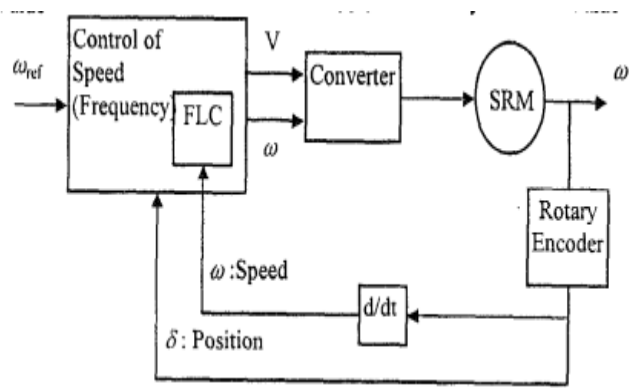

Figure.4 Fuzzy logic controller

\section{Neuro Fuzzy Control Technique (ANFI)}

In [9] discusses the Neuro-fuzzy control technique for torque ripple minimization. Neuro-fuzzy control technique is the pair of ANN and FLC. Fuzzy controller [9] is used for reducing the torque ripples in SRM. FLC has the drawback it gives the better results to low speed and low frequency only. In this Artificial intelligence [9] incorporates with Fuzzy and Neuro technique has self tuning AI. Compensator produces the output signal which is added to the PI gives the reference signal to the current controller.

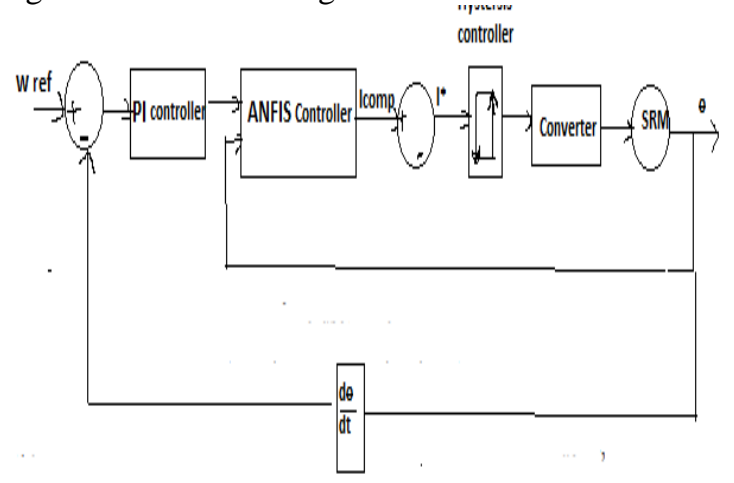

Figure.5 Neuro fuzzy controller

\section{Speed Signal Processing}

In speed signal processing estimated speed signal is processed in embedded [10] controller and correlated to the torque ripple. For implementation of algorithm it requires accurate rotor position information. In [11] discuses the online current profiling method which is used reduce the torque ripples. By using T-I- $\boldsymbol{\theta}$ characteristics method we cannot obtain the precise value of phase current and required rotor position information, to overcome above problem speed signal processing is used. This method has very less complex calculations and it requires less memory storage are the advantages of this method.

\section{Current Chopping Control}

Current control is done with current change closed loop chopping control of the converter. In chopping current Technique the current error is compute from which the switching is generate depending on its connection to the chopping current width [8-9]. The referred current $\mathrm{I}_{\text {ref }}$ would be compared to the motor phase current I as shown

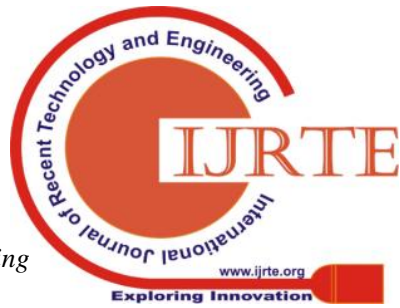


Figure.1. The switching logic for current chopping controller is done as given in equation.

If $\left(I_{\text {reference }}-I\right) \geq \triangle i$ then $U=V_{d c}$

If $\left(I_{\text {reference }}-I\right) \leq i$ then $U=-V_{d c}$

else $U=0$---------(1)

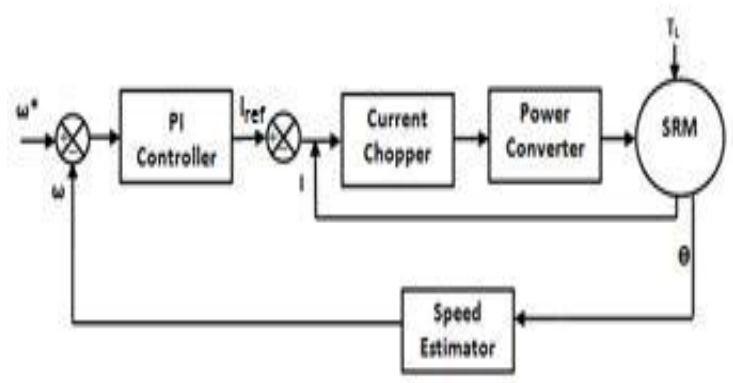

Figure.6 Current chopping controller

\section{Single Pulse Control}

Single pulse control method is used for controlling the motor speed by changing $\theta$ and get optimized system efficiency by adjusting $\theta$ value. Different algorithms are there for getting incremented output by turn on angle $\Delta \theta$. When turn off angle is too large efficiency of system reduces[8], speed increases and torque will affected because current entering into the falling zone of inductance, this problem is overcome by changing turn off angle with phase current and speed . Low acoustic noise obtained by preferable value of excitation angle. That excitation angle has chosen by single pulse control method. In this method the range of surface velocity of commutation angle is compared.

For optimal operation it requires a Single pulse controller with high speed, efficiency and low cost is implemented

At operating point commutation angles are adjusted for giving optimum efficiency[8-12].

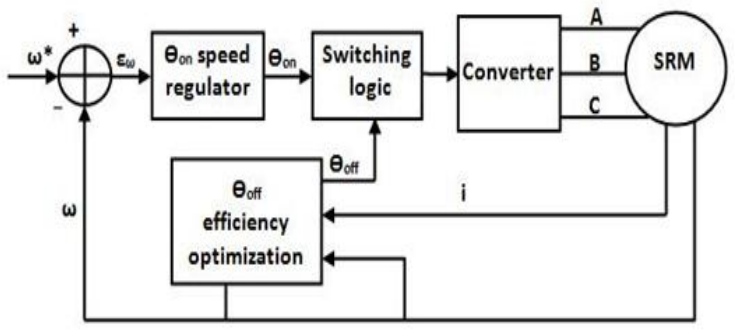

Figure.7 Block diagram of single pulse controller

\section{Instantaneous Torque Control}

Instantaneous torque control gives low torque ripple and quick time response. It compares referred current at each trail in combination with rotor position and reference torque [13]. In this method current and torque are estimated based on all signal patterns and most suitable triggering pulse to find torque value on minimum current is decided [14-15]. It gives high accuracy speed control for SRM. It produces non linear Instantaneous Torque from static torque and look up table.

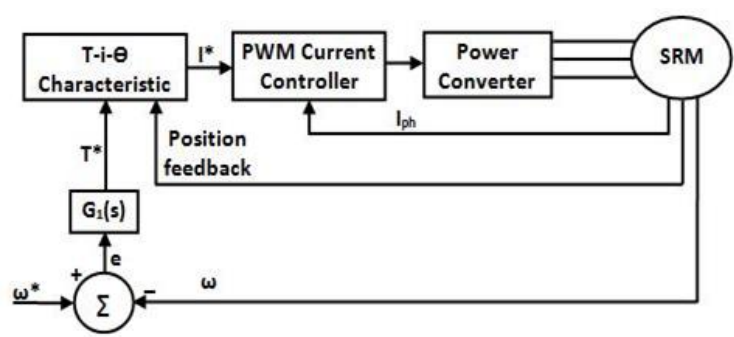

Figure. 8 Block diagram for Instantaneous Torque Control

\section{Torque Sharing Function}

TSF is the best method for the production of constant torque in SRM. The block diagram for torque sharing is shown in figure 9.In this method complete reference torque is divided for each phase and this torque is compared with rotor position[15-19].

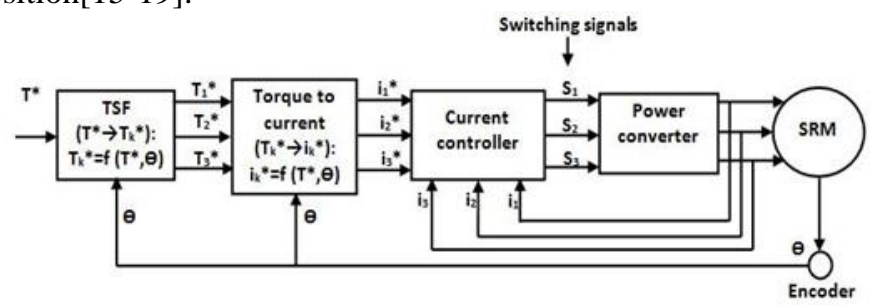

\section{Direct Instantaneous torque control}

Minimization of torque ripples using Direct Instantaneous Torque Control strategies for SRM is proposed [20-23]. Compared to the conventional control strategies, in DITC torque is the reference control variable instead of current. In this method the switching signals are directly produced by comparing the actual torque and reference torque using hysteresis controller as shown in Figureure.8.

\section{CONCLUSION}

This paper has presented a comprehensive technology review of various control strategies on torque ripple minimization for switched reluctance motor drive. Of the various techniques implemented in the past literatures, it is inferred that adaptive fuzzy Neural and speed signal processing techniques have shown significant impact on reduction of torque ripples in SRM 


\section{CONCLUSION}

\begin{tabular}{|l|l|l|l|l|l|}
\hline SI.No. & Control Strategy & $\begin{array}{l}\text { Torque } \\
\text { ripple } \\
\text { speed } \\
\text { operation }\end{array}$ & $\begin{array}{l}\text { Rate } \\
\text { complexity }\end{array}$ & Dynamics \\
\hline 1 & Digital PWM current controller & Low & High & Medium & Fast \\
\hline & & & & & \\
\hline 2 & Fuzzy adaptive direct torque control & Low & Low & Low & Medium \\
\hline 3 & Fuzzy logic controller & Low & Low & Medium & High \\
\hline 4 & Neuro -fuzzy control technique & Low & Medium & High & Medium \\
\hline 5 & Speed signal processing & High & Low & High & Medium \\
\hline 6 & Current Chopping Control method (CCC) & High & Low & High & Medium \\
\hline 7 & Single Pulse Control method (SPC) & High & High & High & Medium \\
\hline 8 & Instantaneous Torque Control (ITC) & Medium & Low & Medium & Low \\
\hline 9 & Torque sharing function & Medium & Low & Medium & Low \\
\hline 10 & $\begin{array}{l}\text { Direct Instantaneous Torque Control method } \\
\text { (DITC) }\end{array}$ & Low & Low & Low & Fast \\
\hline
\end{tabular}

12. Donald $\boldsymbol{S}$. Reay," CMAC And B-Spline Neural Networksapplied To Switched Reluctance Motor Torque Estimation And Control",

\section{REFERENCES}

1. Fei Peng, Student Member, IEEE, Jin Ye, Member, IEEE, And Ali Emadi, Fellow, IEEE." A Digital PWM Current Controller For Switched Reluctance Motor Drives". IEEE Transactions On Power Electronics, Vol. 31,NO.10,October2016, https://doi.org /10.1109/ITEC.2014.68651765

2. Sofiane Fahas, Hoang Le-Huy, Innocent Kamwa," Fuzzy Direct Adaptive Direct Torque Control Of Switched Reluctance Motors", 978-1-5090-3474-1/16/\$31.00@2016IEEE.https://doi.org 10.1109/IECOON.2016. 7793942

3. Dr. E. V. C. Sekhara Rao, "Torque Ripple Minimization Of A Switched Reluctance Motor Using Fuzzy Logic Control”. International Journal On Recent And Innovation Trends In Computing And Communication https://doi.org/10.1007/s13198-014-0232-7

4. Lee, B.S., H.K. Bae, P. Vijayraghavan, And R. Krishnan, "Design Of A Linear Switchedreluctance Machine", Conference. Rec. IEEE Ind. Appl. Soc. Ann. Mtg.,Oct. 2267-2274,1999. https://doi.org /10.1109/28.887208

5. L. O. P. Henriques, L. G. B. Rolim, W. I. Suemitsu, P. J. Costa. Branco Reluctance Drive Using A Neuro-Fuzzy Control Technique".

6. Yang Yangguo, Qishualgma, Weiye "Comparitive Study On Torque Ripple Suppression Method Of Three-Phase 6/4 Switched Reluctance Motor"978/1-5090-1087-5/16@2016IEEE_OCT10/12,2016). https://doi.org /10.1109/AUS.2016.7748074.

7. Iqbal Husain, Mehrdad Ehsani,"Torque Ripple Minimization In Switched Reluctance motor Drives By PWM Current Control",0-7803-1456-5/94 \$4.00 01994 IEEE. . https://doi.org /10.1109/63.484420.

8. J. I. Zhi-cheng, H. Xue, "Study on Modeling and Simulation of Switched Reluctance Motor Control System Based on Matlab," Journal. System Simulation, vol. 17, no.4, pp. 1015-1021, April. 2005. . https://doi.org /10.1109/IEC.2004.1248564.

9. J. Lili, Chen Hao, "Nonlinear modeling and simulation of switched reluctance motor drive system based on Matlab," Journal. Southeast

10. J.M.Stephenson, A.Hughes And R.Mann," Torque Ripple Minimisation In A Switched Reluctancemotor By Optimum Harmonic Current Injection" IEE Proc.-Electr. Power Appl.. Vol. 148, No. 4, J U LY 2001 . https://doi.org /10.1109/ip-epa:20010480.

11. Jaehyuck Kim, R. Krishnan, "High Efficiency Single-Pulse Controlled Switched Reluctance Motor Drive for High Speed (48k rpm) Application: Analysis, Design and Experimental Verification", in proc.IAS Conf, pp. 1-8, 2008. . https://doi.org /10.1109/08IAS.2008.196. And J. A. Dente, "Torque Ripple Minimization Of Switched https://doi.org /10.1.1.31.3963. University of China (Natural Science Edition), vol. 34, pp. 149-154

13. Zheng Hongtaols, Lin Feng, Liu Liangen, Jiang Jingping, Xu Dehong," Torque Ripple Minimization In Switched Reluctance Motors Using Fuzzy-Neural Network Inverse Learning Control" 0-7803-7885-7/03/S17.00 $02003 \quad$ IEEE. . https://doi.org /10.1109/PEDS.2003.1283148.

14. Natan Tzvi Shaked And Raul Rabinovici, Senior Member, IEEE," New Procedures For Minimizing The Torque Ripple in Switched Reluctance Motors By Optimizing the Phase" . https://doi.org /10.1109/TMAG.2004.843311

15. 'Isma'Il A־Girman, Aleksandar M. Stankovic, Gilead Tadmor," Adaptive Torque-Ripple Minimization In Switched Reluctance Motors", Ieee Transactions On Industrial Electronics, Vol. 48, No. 3 , June 2001. . https://doi.org /10.1109/28.753642.

16. Y. Jin, B. Bilgin, "An Extended-Speed Low-Ripple Torque Control of Switched Reluctance Motor Drives”, IEEE Trans. Power Electronics, vol. 30, no. 3, March. 2015.

17. V. P. Vujiccic, "Minimization of torque ripple and copper losses in switched reluctance drive," IEEE Trans. Power Electron, vol. 27, no.1, pp. 388-399, Jan. 2012

18. D. H. Lee, Z. G. Lee, and J. W. Ahn, "A simple nonlinear logical torque sharing function for low-torque ripple SR Drive," IEEE Trans. Ind Electron, vol. 56, no. 8, pp. 3021-3028, Aug. 2009. . https://doi.org /10.1109/TIE.2009.2024661.

19. S. K. Sahoo, S. K. Panda, and J. Xu, "Indirect torque control of switched reluctance motors using iterative learning control," IEEE Trans. Power Electron, vol. 20, no. 1, pp. 200-208, Jan. 2005. https://doi.org /10.1109/TPEL2004.839807.

20. P.Chancharoensook, "Direct Instantaneous Torque Control of a Four-Phase Switched Reluctance Motor," in proc. PEDS Conf, pp. 770-777, 2009.

21. Castro, P. Andrada, "Minimization of Torque Ripple in Switched Reluctance Motor Drives Using an Enhanced Direct Instantaneous Torque Control," in proc. ICEM Conf, pp. 1021-1026, 2012. https://doi.org /10.1109/ICEMACH.2012.6350001

22. Qionghua Zhan, Jianbo Sun, “A Fixed-Frequency Direct Instantaneous Torque Control Method of Switched Reluctance Motor Contributing to Low Vibration and Acoustic Noise", IEEE Trans. Industrial Electronics, pp. 1580-1585, 2006.

23. C. R. Neuhaus, N. H. Fueng warodsakul, "Predictive PWM-based Direct Instantaneous Torque Control of Switched Reluctance Drives", in proc. PESC Conf, pp. 1-7, 2006.

24. Rakesh Mitra, Yilmaz Sozer, "Torque Ripple Minimization Of Switched Reluctance Motors Through Speed Signal Processing".978-1-4799-5776-7/14/\$31.00 @2014 IEEE https://doi.org /10.1109/ECCE.2014.6953577. 